\title{
Fremden- und Weltstadt Dresden? Ambition und Realität im Deutschen Kaiserreich
}

\author{
von
}

NADINE ZIMMERLI

Eine Werbung zur Ersten Internationalen Hygieneausstellung in Dresden bezeichnete die sächsische Hauptstadt 1911 in großen schwarzen Worten als: Dresden - bevorzugte Fremdenstadt Deutschlands. Die Anzeige vermerkte auch, dass Dresden vorzüglich geeignet zum dauernden Aufenthalt wäre. ${ }^{1}$ Die Ausstellung brachte insgesamt fast fünf Millionen Besucher in die Stadt und zumindest dieser Zeitungsanzeige nach hießen die Dresdner die Fremden nicht nur während der Ausstellung willkommen, sondern luden sie gezielt ein, sich dauerhaft niederzulassen. Dresdner Zeitungen hatten in der Ausstellung schon Jahre vorher ein großes Potenzial erkannt, wie ein Artikel 1908 in den „Dresdner Neuesten Nachrichten" spekulierte: Es handelt sich um ein Unternebmen, das geeignet ist, die Augen der ganzen Welt auf Dresden zu lenken, um eine Ausstellung, wie sie hier noch nie und sonst wobl nur in Weltstädten, wie Paris, London, Berlin und Wien, geseben worden ist. [...] Gelingt der Plan auch nur einigermaßen, so wird Dresden nicht nur für die Dauer der Ausstellung eine Weltstadt sein. ${ }^{2}$ Anfang des 20. Jahrhunderts stellte sich Dresden als die anziehendste Stadt Deutschlands mit internationalem Charakter dar, rief fremde Besucher auf, sich auch permanent anzusiedeln, und sah in all dem eine Chance, in die Riege der Weltstädte aufzusteigen.

Der „Verein zur Förderung Dresdens und des Fremdenverkehrs“ ging sogar noch einen Schritt weiter und sah Dresden schon 1910 als direkte Weltstadt an, auch ohne die Hygieneausstellung. Er nannte die Stadt in einer englischsprachigen Broschüre literally a cosmopolitan bub, also einen wortwörtlich weltstädtischen Drehpunkt. Weiter hieß es: the foreigner, indeed, is very much a privileged person in Dresden (Der Ausländer ist in Dresden wirklich eine sehr privilegierte Person). ${ }^{3}$ Diese Zitate weisen darauf hin, dass Dresden zur Zeit des Kaiserreiches gezielt eine Identität als ,Fremdenstadt' aufbaute, die manchen Dresdnern dann sogar Anlass gab, ihre Stadt als, Weltstadt' zu betrachten. Unter dem Begriff Fremdenstadt verstanden die Dresdner auch nicht nur eine Touristenmetropole, sondern sie sahen ihre Stadt als geradezu geprägt von staatsfremden Ausländern. Um nur

1 Werbung gefunden im Sächsischen Staatsarchiv - Hauptstaatsarchiv Dresden, Zeitungsausschnittsammlung, Z/Bd. 1, I. Internationale Hygiene-Ausstellung, Dresden 1911.

2 Dresdner Neueste Nachrichten, 21. Mai 1908, S. 2.

3 Dresden. The Jewel of Northern Europe, hrsg. vom Verein zur Förderung Dresdens und des Fremdenverkehrs, Dresden 1910, S. 5, 23. 


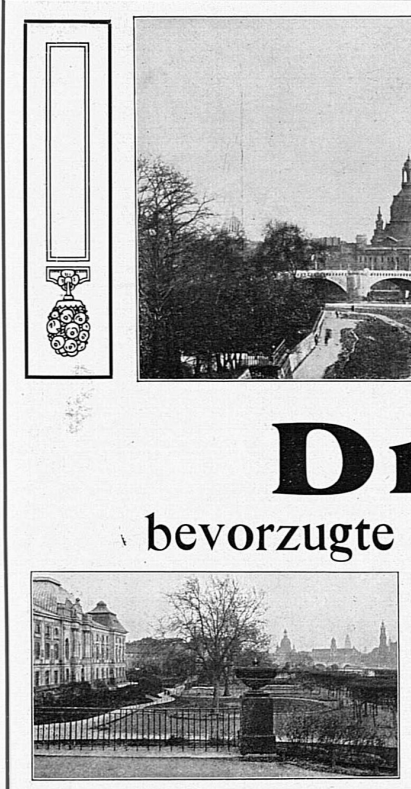

Japanisches Palais.

Nadine Zimmerli

Dresden $\begin{aligned} & \text { birgt die Königliche Gemäldegalerie mit der weltberühmten Sixtinischen Madonna und einen } \\ & \text { unvergleichlichen Rembrandtsaal, das Grüne Gewölbe mit Kunstschätzen und Juwelen von unschätz- }\end{aligned}$ barem Werte. dil. Das Johanneum umfasst die einzigartige Porzellansammlung und Deutschlands grösstes Waffenmuseum.

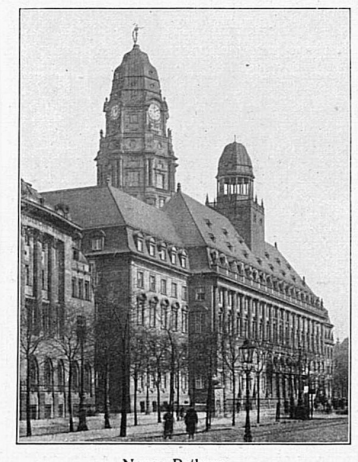

Dresden hat eine weltberühmte Dresden Königl. Hofoper: Richard Strauss-Ur-Aufführungen: Elektra - Salome Rosenkavalier; regelmässig Wagner-Aufführungen usw. Das Königl. Schauspielhaus hat einen hervorragenden modernen und klassischen Spielplan. Im Winter 12 Sinfonie-Konzerte der Königlichen Kapelle und zahlreiche Kammermusikabende.

Dresden hat weltberühmte Denk. Zwinger - Japanisches Palais - Frauenkirche Katholische Hofkirche. Berühmt ist das DresdenerMeissner Porzellan der Königlichen Manufaktur.

Dresden $\begin{aligned} & \text { bietet Sport aller Art: Golf } \\ & \text { Pferderennen-Ballonsport }\end{aligned}$ Ruderregatten - Lawntennis - Fussball - Polo.

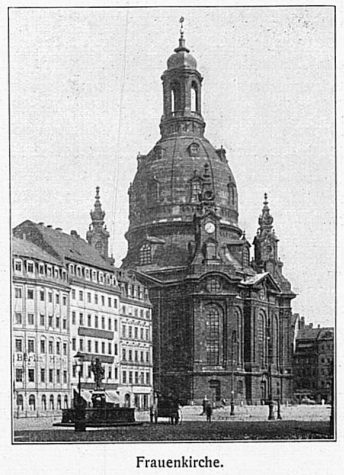

Dresden ist vorzüglich geeignet zum dauernden Aufenthalt. Vorzügliche neue Hotels, zahlreiche Pensionen, DreSUEn Technische Hochschule, Tierärztliche Hochschule, Kunst- und Musikschulen. Höhere Schulen aller Art. Alljährlich grosse Ausstellungen im städtischen Ausstellungspalast.

Internationale Hygiene-Ausstellung Dresden 1911.

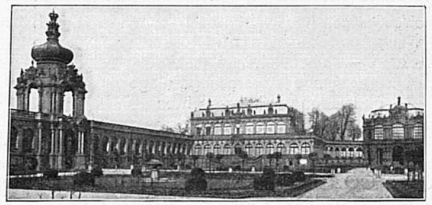

Zwinger.

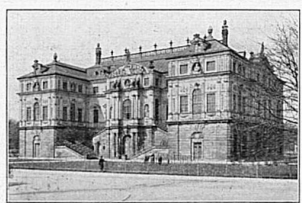

Palais im Grossen Garten.

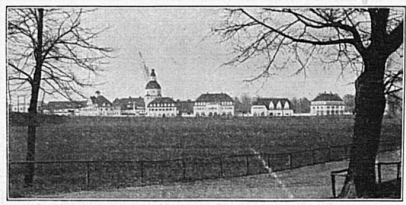

Schlachthof.

Abb. 1: Werbeplakat zur Ersten Internationalen Hygieneausstellung in Dresden 1911. 
ein weiteres Beispiel aufzuführen, auch die Organisatoren der Hygieneausstellung übersetzten Fremdenstadt als international city of foreigners, also als ein Label direkt auf Ausländer und nicht auf deutsche Besucher bezogen. ${ }^{4}$

Dieser Ruf als ,international city of foreigners' war nicht unbegründet. Es ist bekannt, dass Dresden eine der deutschen Großstädte mit dem höchsten Ausländeranteil unter den Einwohnern war. Er schwankte im Kaiserreich zwischen vier und sechs Prozent und lag damit doppelt bis dreifach so hoch wie der Anteil in Leipzig oder Berlin. ${ }^{5}$ Dresdens Anziehungskraft auf Touristen ist ebenso bekannt. Zum Beispiel stieg die Zahl der registrierten Besucher von bereits über $321000 \mathrm{im}$ Jahr 1899 auf mehr als 433000 im Jahr 1909. Um die Jahrhundertwende gab es schon mehr als 3000 Hotels und Gaststätten. ${ }^{6}$

Dennoch überrascht, dass die obigen Zitate bezeugen, dass Dresden während der Zeit des Kaiserreiches gezielt und bewusst diese Identität als internationale Fremdenstadt auf- und ausbaute, dass Ausländer zum ständigen Aufenthalt angehalten worden und dass diese urbane Identität Erwähnungen von Weltstadt/Kosmopolitismus im positiven Sinne bis kurz vor dem Ausbruch des Ersten Weltkrieges mit sich zog. Es lohnt sich genau zu historisieren, was die Dresdner damals unter den Begriffen ,Fremdenstadt' und ,Weltstadt/Kosmopolitismus'verstanden haben. Im Gegensatz zum Dresdner Beispiel verbanden andere Fremdenverkehrsvereine im Kaiserreich Tourismus nämlich mit Heimatverständnis und beförderten dadurch gezielt die Entstehung einer nationalen Identität, wie die Arbeiten Rudy Koshars, Celia Applegates, Alon Confinos und Caitlin Murdocks gezeigt haben. ${ }^{7}$

4 ,City of Strangers` wäre die direktere Übersetzung, doch die Dresdner wollten wohl gezielt den internationalen Charakter der Stadt unterstreichen. Die Organisatoren der internationalen Hygieneausstellung druckten dieselben Werbematerialien sowohl auf Deutsch als auch auf Englisch, und Fremdenstadt erscheint als international city of foreigners in diesen Materialien. Das deutsche Original befindet sich in der Sächsischen Landesbibliothek - Staats- und Universitätsbibliothek Dresden (im Folgenden: SLUB), Internationale Hygiene-Ausstellung, Dresden 1911, Mai bis Oktober, S. 7, Signatur H. Sax. G 126 SC. Das englische Pendant befindet sich in der University of WisconsinMadison Ebling Library for the Health Sciences, International Hygiene Exhibition, Dresden 1911, S. 7. Hinzu kommt noch, dass der Fremdenverkehrsverein 1908 mehrere Werbungen schaltete und in diesen Dresden auf Deutsch als die vornehmste Fremdenstadt Deutschlands und auf Englisch als the most attractive City for Foreigners verkaufte. Vgl. Dreiunddreissigster Rechenschafts-Bericht des Vereins zur Förderung Dresdens und des Fremdenverkehrs in Dresden, erstattet auf die Zeit vom 1. April 1908 bis 31. März 1909, Dresden 1909, S. 11-13.

5 Vgl. Holger Starke/Uwe John (Hg.), Geschichte der Stadt Dresden, Bd. 3: Von der Reichsgründung bis zur Gegenwart, Stuttgart 2006, S. 236 f.

6 Dresden. Die Geschichte der Stadt, hrsg. vom Dresdner Geschichtsverein, Hamburg 2002, S. 194. Auch Andrea Dietrich, Die Entwicklung der Stadt Dresden zu einer Tourismusmetropole von den Anfängen bis zum Vorabend des ersten Weltkrieges, 2 Bände, Diss. Universität Leipzig 1992.

7 Vgl. Rudy Koshar, ,What Ought to be Seen'. Tourists' Guidebooks and National Identities in Modern Germany and Europe, in: Journal of Contemporary History 33 (1998), Nr. 3, S. 323-340; Ders., German Travel Cultures, New York 2000; Celia 
Generell verbindet die Geschichtswissenschaft die Zeit des Kaiserreiches aus gutem Grund mit einem immer stärker werdenden Nationalismus. ${ }^{8}$ Aussagen wie diese aus dem Jahr 1898 waren keine Seltenheit: Cosmopolitismus und Internationalismus, das heisst also, was das hassenswerth lächerlichste und antinational blödsinnigste ist, das es gibt. ${ }^{9}$ Zehn Jahre später veröffentlichte der einflussreiche Historiker Friedrich Meinecke sein Buch Weltbürgertum und Nationstaat. In ihm argumentierte er, dass der Kosmopolitismus des 18. Jahrhunderts im 19. Jahrhundert untragbar geworden war und vom Nationalgedanken ersetzt werden musste, damit sich Staaten wie das vereinigte Deutschland entfalten konnten. ${ }^{10}$ Hinzu kommt noch, dass Historiker Dresden schon seit langem als eine der konservativsten und national-gesinntesten deutschen Städte betrachten. Immerhin schrieb Julius Langbehn die hochnationalistische Abhandlung „Rembrandt als Erzieher" in der Stadt, Oberbürgermeister Gustav Otto Beutler (von 1895 bis $1915 \mathrm{im} \mathrm{Amt)} \mathrm{trat} \mathrm{häufig} \mathrm{bei} \mathrm{Veranstaltungen} \mathrm{von} \mathrm{konservativen} \mathrm{und} \mathrm{nationalisti-}$ schen Vereinigungen, die die Stadtverwaltung auch oft finanziell unterstützte, als Eröffnungsredner, Schirmherr oder Ehrengast in Erscheinung, und Historiker des Alldeutschen Verbandes haben mehrmals gezeigt, dass die Dresdner Ortsgruppe eine der aktivsten im ganzen Kaiserreich war. ${ }^{11}$ Wie also ist Dresdens Ambition

Applegate, A Nation of Provincials. The German Idea of Heimat, Berkeley 1990; Alon Confino, The Nation as a Local Metaphor. Württemberg, Imperial Germany, and National Memory, 1871-1918, Chapel Hill 1997; Caitlin E. Murdock, Tourist Landscapes and Regional Identities in Saxony, 1878-1938, in: Central European History 40 (2007), S. 589-621.

8 Vgl. z. B. Dieter Langewiesche (Hg.), Nation, Nationalismus, Nationalstaat in Deutschland und Europa (Beck'sche Reihe 1399), München 2000; Peter Walkenhorst, Nation - Volk - Rasse. Radikaler Nationalismus im Deutschen Kaiserreich 1890-1914 (Kritische Studien zur Geschichtswissenschaft 176), Göttingen 2007.

9 Bertha von Suttners Friedensbewegung veröffentlichte diese Aussage als Paradebeispiel um zu zeigen, wie Nationalisten die Friedensbewegung einschätzten: Weltbürgerthum im Urtheil des Nationalisten, in: Die Waffen nieder! Monatsschrift zur Förderung der Friedensbewegung 7 (1898), S. 157.

10 Friedrich Meinecke, Weltbürgertum und Nationalstaat. Studien zur Genesis des deutschen Nationalstaates, München/Berlin 1908.

11 Vgl. Jürgen Paul, Der „Rembrandtdeutsche“ in Dresden, in: Dresdner Hefte 57 (1999), S. 11; und besonders Gerald Kolditz, Politische Vereine und Parteien, in: Starke/John, Geschichte der Stadt Dresden (wie Anm. 5), Bd. 3, S. 50-58. Kolditz fasst Dresdens politisches Klima folgendermaßen zusammen: „In den zwei Jahrzehnten vor Ausbruch des Weltkrieges dominierte im öffentlichen Leben Dresdens ein deutschnationales, konservativ-monarchistisches Klima" (S. 53). Zum Alldeutschen Verband in Dresden vgl. Roger Chickering, We Men Who Feel Most German. A Cultural Study of the Pan-German League, 1886-1914, Boston 1984. Auch Gerald Kolditz, Rolle und Wirksamkeit des Alldeutschen Verbandes in Dresden zwischen 1895 und 1918. Ein Beitrag zum bürgerlichen Vereinsleben der nationalistischen Kräfte in der wilhelminischen Ära des deutschen Kaiserreiches, Diss. TU Dresden 1994; Ders., Der alldeutsche Verband in Dresden. Antitschechische Aktivitäten zwischen 1895 und 1914, in: Rainer Aurig/Steffen Herzog/Simone Lässig (Hg.), Landesgeschichte in Sachsen. Tradition und Innovation (Studien zur Regionalgeschichte 10), Bielefeld 1997, S. 235248. 
zur internationalen Fremden- und gar Weltstadt, und beides positiv gemeint, zu genau diesem Zeitpunkt einzuordnen?

\section{Dresdner Selbstzengnisse}

Zeitgenössische Dresdner Selbstzeugnisse geben einen Anhaltspunkt. Ein gewisser Victor von Hüben veröffentlichte 1908 einen Überblick Dresdens, „Mein Dresden lob ich mir“, der alle Facetten der Stadt beleuchtete. Die Beschreibung begann mit Lobreden auf lokale Sehenswürdigkeiten und einem Besuch des Stadtmuseums, welches sich vorzüglich als Näbrboden der Freude an der Heimat und der Liebe zur Heimat eignete. ${ }^{12}$ Dann beschrieb Hüben nationale Feste, da der Dresdner, wie überhaupt der Sachse [...] in erster Linie deutsch denkt und deutsch füblt. ${ }^{13}$ Doch damit nicht genug. Die Beschreibung kulminierte in einem Überblick der ansässigen anglo-amerikanischen Kolonie und wies stolz darauf hin, dass der britische Humorist Jerome K. Jerome, der englische Mark Twain, wie Hüben ihn bezeichnete, die Stadt die anziehendste, die Deutschland aufzuweisen habe, genannt hatte. ${ }^{14}$ „Mein Dresden lob ich mir“ skizzierte gleichzeitig Dresdens lokale, nationale und internationale Dimensionen und gab allen drei Registern gleichwertige Aufmerksamkeit. Anders ausgedrückt war Dresden dank seiner lokalen, nationalen und internationalen Sehenswürdigkeiten, Feste und Anwohner bedeutsam, ohne dass sich die drei Facetten im Weg stünden. Dresdens Ambitionen als ausländerfreundliche Weltstadt um 1900 standen im Urteil zeitgenössischer Autoren also in keinem Widerspruch zu Dresden als sächsischer Hauptstadt oder gar als nationaler Hochburg. Alle drei Register - lokal, national, international - bildeten parallele, sogar komplementäre Facetten.

Noch wichtiger ist die Erkenntnis, dass es kein Zufall war, dass der internationale Teil von Hübens Beschreibung die anglo-amerikanische Kolonie in den Vordergrund stellte. Genau so wenig war es ein Zufall, dass der Fremdenverkehrsverein Dresden als cosmopolitan bub auf Englisch in einer gänzlich englischsprachigen Broschüre gepriesen hatte. Wie diese zwei Beispiele schon andeuten, bezogen sich Dresdens Identität als internationale Fremdenstadt und Ambitionen zur Weltstadt an allererster Stelle auf die zahlreichen britischen und amerikanischen Besucher und anglo-amerikanischen Einwohner der Stadt. Wie eine weitere Kommentatorin zu Dresdens Charakter 1907 bemerkte: Bedeutend mehr, als in einer anderen großen Stadt fällt in Dresden das Kontingent seiner Fremden auf, und von diesen bei weitem am meisten die Engländer und Amerikaner. Das Bild langer Engländer, bübscher amerikanischer boys und schlanker eleganter Frauen beider

12 Victor von HüBen, Mein Dresden lob ich mir. Federzeichnungen, Dresden 2[1908], S. 19.

13 Ebd., S. 25 f.

14 Ebd., S. 58. 
Rassen gehört fast unzertrennlich zu dem Dresdener Straßenbild. ${ }^{15}$ Dresdens angelsächsischen Besucher und Einwohner bildeten einen wichtigen Teil, wenn nicht sogar den Kern der städtischen Identität, eng mit dem Straßenbild der Stadt verbunden. Der eigentlich weitläufige Begriff ,Fremde` war hier auf diese eleganten englischsprachigen Ausländer begrenzt.

Ein Artikel im „Dresdner Anzeiger“ zu „Dresden als Fremdenstadt“ ein Jahr später stellte diese Verflechtung noch stärker in den Vordergrund. Obwohl der Kopfzeile nach der Artikel scheinbar alle Touristen und Ausländer in Dresden behandelte, so verknüpfte er Dresdens Internationalität fest mit der Anwesenheit anglofoner Touristen und Einwohner. Der Artikel sprach von einem Strom der Fremden, der regelrecht durch Dresdens Straßen wallt, doch auch ohne ihn zeige die Stadt internationale Züge: Zwar hat Dresden schon an sich etwas von internationalem Gepraege. Namentlich das anglo-amerikanische Element ist stark vertreten; im Schweizerviertel scheinen die englischen Firmenschilder der Pharmacy, des hairdresser, des candy-Store die deutschen zu verdrängen. Der ganze Artikel, nicht nur dieser eine Satz, war mit englischen Vokabeln durchsetzt, die Wohlstand und Muße ausdrückten, unter anderem hairdresser, fashionable, globetrotter und five o'clock tea. ${ }^{16}$ Dresdner verbanden das permanente internationale Antlitz ihrer Stadt mit den Anglo-Amerikanern, die unter ihnen lebten. Doch damit nicht genug. Sie hofften auch, dass weitere Besucher dank Dresdens Vorzügen die Stadt zur Wahlheimat erküren. Der letzte Satz des Artikels ist für diese Haltung bezeichnend: Bleibe bei uns, du Fremdling!17 Dresdens Selbstverständnis als Fremdenstadt war eng mit der ansässigen anglo-amerikanischen Kolonie verbunden, und es war ausgesprochenes Ziel, weitere ausländische Einwohner dieser Art für die Stadt zu gewinnen.

Dresden war Anglo-Amerikanern schon seit dem 18. Jahrhundert als eine Station auf der aristokratischen und später der bürgerlichen Grand Tour (Bildungsreise) ein Begriff. ${ }^{18}$ Bereits 1792 gab es Reiseberichte, die vermerkten, dass sich zahlreiche Engländer in Dresden niedergelassen hatten. ${ }^{19}$ Im Jahre 1841 gründeten die ansässigen Engländer dann eine anglikanische Gemeinde, und es gab 1845 ungefähr 200 englische Einwohner. ${ }^{20}$ Ihnen schlossen sich nach und nach die Amerikaner an, und im November 1858 gründeten achtzehn Briten und Amerika-

15 Eva Gräfin von Baudissin, Die Amerikanische und Englische Kolonie, in: Freiherr von Schlicht [Wolf Graf von Baudissin] (Hg.), Dresden und die Dresdener. Ein lustiges Vademecum, Dresden/Leipzig 1907, S. 62.

16 Erich O. Moeller, Dresden als Fremdenstadt, in: Dresdner Anzeiger, 2. August 1908, S. 7.

17 Ebd.

18 Vgl. Dietrich, Entwicklung der Stadt Dresden (wie Anm. 6), Bd. 1, S. 12.

19 Vgl. Brian Dolan, Ladies of the Grand Tour. British Women in Pursuit of Enlightenment and Adventure in Eighteenth-Century Europe, New York 2001, S. 105.

20 Vgl. Paul W. Schniewind, Anglicans in Germany. A History of Anglican Chaplaincies in Germany until 1945, Darmstadt 1988, S. 89. 
ner den Anglo-American Club in Dresden, den ersten dieser Art in ganz Europa. ${ }^{21}$ Als Newell Sill Jenkins, ein amerikanischer Zahnarzt, der bis zum Ersten Weltkrieg in Dresden verweilte, 1866 in die Stadt zog, fand er schon eine beträchtliche englische und amerikanische Kolonie vor. ${ }^{22}$ Im Jahr 1869 wurde dann die anglikanische Kirche All Saints eingeweiht, und es gründete sich im selben Jahr auch formell eine amerikanisch-episkopalische Kirchengemeinde. ${ }^{23}$

Jedoch entfalteten diese englischsprachigen Ausländer erst nach der Reichsgründung 1871 ihre volle Stärke - z. B. erschien die erste englische Zeitung Dresdens, „The Stranger's Guide (Dresden Advertiser)“, in diesem Jahr - und erlangten auch eine völlig neue Bedeutung für ihre Wahlheimat. ${ }^{24}$ In den 1870 er-Jahren begannen Dresdner, diese Ausländer direkt zu werben und im Dienste der Fremdenstadtidentität für die ständige Niederlassung zu rekrutieren. Laut meiner Erkenntnis waren wohlhabende Ausländer in der Stadt, besonders jene, die sich permanent niederließen, ein wichtiges Zeichen, dass Dresden selbst nach der Reichsgründung und dem damit verbundenen Verlust politischer Unabhängigkeit und der unangefochtenen Vormachtstellung Berlins trotzdem noch eine bedeutende Stadt war.

Anders ausgedrückt befürchteten die Dresdner nach der Reichsgründung 1871, dass die Stadt ihren internationalen Status verlieren würde, und das Umwerben wohlhabender Ausländer war eine kommunale Strategie, um das Abrutschen in die befürchtete Mittelmäßigkeit zu verhindern. Wie die Leiterin einer Pension für englischsprachige Mädchen 1896 prägnant feststellte: Obne die Ausländer wäre Dresden ein Dorf. ${ }^{25}$ Die englischsprachigen Ausländer sahen die Sache ge-

21 Vgl. die Festreden zum Anlass des Gründungsjahres des Klubs, gedruckt im Stranger's Guide to Dresden, 5. November 1898, S. 1-3; auch HüBEN, Mein Dresden lob ich mir (wie Anm. 12), S. 59.

22 Newell Sill Jenkins, Reminiscences of Newell Sill Jenkins, Princeton 1924, S. 90.

$23 \mathrm{Zu}$ den Kirchen vgl. Schniewind, Anglicans in Germany (wie Anm. 20), S. 92. Es gibt eine Handvoll Veröffentlichungen zu den Amerikanern in Sachsen und speziell in Dresden in der ersten Hälfte des 19. Jahrhunderts, u. a. EBERHARD BRÜNING, „Elb-Florenz“ versus „Spree-Athen“. Die amerikanische Bildungselite des 19. Jahrhunderts und ihr Bild von Dresden, in: Amerikastudien/American Studies 36 (1991), Nr. 2, S. 195-208; DERS., Sachsen mit amerikanischen Augen gesehen. Das Sachsenbild amerikanischer Globetrotter im 19. Jahrhundert, in: NASG 67 (1996), S. 109-131; und Ders., Saxony Is a Prosperous and Happy Country. American Views of the Kingdom of Saxony in the Nineteenth Century, in: Thomas Adam/Ruth Gross (Hg.), Traveling between Worlds. German-American Encounters, Arlington 2006, S. 20-50. Vgl. auch Ashley Sides, „That Humane and Advanced Civilization“. Interpreting Americans' Values from Their Praise of Saxony, 1800-1850, in: Thomas Adam/Nils H. Roemer (Hg.), Crossing the Atlantic. Travel and Travel Writing in Modern Times, Arlington 2011, S. 11-49. Auch von Bedeutung ist die Primärquelle Thomas Adam/Gisela Mettele (Hg.), Two Boston Brahmins in Goethe's Germany. The Travel Journals of Anna and George Ticknor, Lanham 2009.

24 Die Geschichte der Zeitung ist zu finden in: Stranger's Guide to Dresden, 5. März 1910, S. 1.

25 So festgehalten von einer englischen Schülerin, die 1896 in Dresden verweilte: The Women's Library, London Metropolitan University, Kathleen D’Olier to Mama, 2. Februar 1896, Papers of Kathleen D'Olier Courtney, 7KDC/A/2. 
nauso: Lass es der anglo-amerikanischen Kolonie eines feinen Morgens in den Sinn kommen ibre Zelte abzubrechen und umzuziehen und Dresden würde sofort auf das Level einer mittelmäßigen Landstadt herabsinken. ${ }^{26}$ Die anglo-amerikanische Kolonie gab der Stadt ihr internationales Ansehen und generelle Bedeutung. Ohne sie war Dresden nur Mittelmaß.

Wegen der Angst, dass Ausländer Dresden nicht weiter frequentieren und die Stadt in die internationale Bedeutungslosigkeit versinken würde, war eine 1877 erschienene „Statistik der in Dresden sich aufhaltenden Fremden“ in Auftrag gegeben worden: Die über die Abnabme des Dresdner Fremdenverkehrs laut gewordenen Befürchtungen gaben dem statistischen Bureau Veranlassung, der Statistik des Dresdner Fremdenwesens, bei Bearbeitung des 1875 er Volkszählungsmaterials, eine besondere Berücksichtigung zu Theil werden zu lassen. ${ }^{27}$ Diese Fremdenstudie war die erste Bestrebung städtischer Behörden, die ausländischen Bewohner Dresdens systematisch zu erfassen, zu klassifizieren und deren kommunale Bedeutung zu untersuchen. Erleichtert stellte man jedoch fest, dass Dresden nichts an internationaler Anziehungskraft verloren hatte. Die Anzahl der ansässigen Ausländer hatte sich stetig vermehrt, von 4658 im Jahr 1867 auf $6250 \mathrm{im} \mathrm{Jahr} 1871$ und schließlich auf 8026 im Jahr 1875.28 Wie die Statistiker mehrfach betonten, waren dahin gehende Ängste also unbegründet. Bedeutender ist jedoch, dass die Fremdenstudie weitläufige Einblicke in die Lebenswelten der Ausländer in Dresden und auch in die Gedankenwelt und Prioritäten der städtischen Beamten gewährt. Kurz zusammengefasst beinhaltete die Studie eine eingehende statistische Untersuchung aller ansässigen Ausländer nach Herkunftsland und wirtschaftlichem Status (in Lohn stehend oder konsumtiv) sowie ausführliche Kommentare zur Bedeutung verschiedener Gruppen für die Stadt Dresden. Das Statistische Bureau stellte fest, dass die Mehrheit der Österreicher zum armen, unselbstständigen Teil der Gesellschaft gehörte, während die meisten Russen, Briten und Amerikaner wohlhabende und wirtschaftlich unabhängige Einwohner waren, die ihr Geld in Dresden ausgaben, anstelle es vor Ort zu verdienen. ${ }^{29}$

26 Im Original hieß es: Dresden is far from being a large metropolis, though the good Dresdeners are very zealously indulging in big, imposing expressions proclaiming the greatness and importance of the town. Let the Anglo-American Colony some fine morning make up its mind to shift to some other place, and Dresden would at once sink to the level of a middling country town interesting chiefly for its Gallery of Pictures and the attractiveness of its vicinity. Vgl. A Guide to Dresden, its Buildings, Institutions and Environs. With a Plan of the Town, Dresden o. J., S. 29. Dieser Führer erschien meiner Meinung nach zwischen 1873 und 1878. Er druckte einen Artikel, der im März 1873 in der amerikanischen Zeitschrift „Harper’s New Monthly Magazine“ erschienen war, in Auszügen nach und berichtete auch, dass das Königliche Hoftheater 1869 abgebrannt war und ein zweites (1878 eröffnet) sich noch in der Bauphase befand.

27 Statistik der in Dresden sich aufhaltenden Fremden, in: R. Jannasch (Hg.), Mittheilungen des Statistischen Bureaus der Stadt Dresden, Nr. 4A, Teil II (im Folgenden: Statistik II), Dresden 1877, S. 73.

Ebd.

Vgl. ebd., S. 73-100, hier 92 f. 
Vielsagenderweise ging das Statistische Bureau nicht weiter auf die wirtschaftlichen Kategorien der Lohnarbeiter ein, zu der die Österreicher gehörten. Dennoch sahen sie deren Präsenz als relativ positiv: Die productive Classe der Fremdenbevölkerung erhöht die wirthschaftliche Kraft der städtischen Bevölkerung, sichert und fördert die Güterproduction und trägt somit dazu bei, die Handels- und Wirthschaftsbilanz Dresdens zu einer günstigen zu gestalten. ${ }^{30}$ Weitaus wichtiger waren für die Statistiker jedoch die wohlhabenden Ausländer, deren wirtschaftliche Möglichkeiten und Aktivitäten sie mit einem fünfseitigen Kommentar deutlich eingehender behandelten und zu folgenden Schlüssen kamen: Die consumptive Classe der Fremdenbevölkerung ist, nach den obigen Ziffern zu urtheilen, ein für Dresdens wirthschaftliche Entwickelung sehr berücksichtigenswerther Faktor. Wie es ein paar Zeilen weiter hieß: Offenbar ist es für die wirthschaftliche Prosperität der städtischen Einwohnerschaft in hohem Grade wichtig, dass ibr die durch eine wohlhabende, consumtionsfähige Fremdenbevölkerung zugeführte Einkommensquelle erbalten bleibe. ${ }^{31}$

Die Fremdenstudie hob also die wirtschaftliche Bedeutung der 2396 russischen, britischen und amerikanischen Einwohner Dresdens besonders hervor und ging nur kurz auf die der 4407 Österreicher ein, die vornehmlich der Arbeiterklasse angehörten. Erstere galt es zu rekrutieren, die Anwesenheit Letzterer nahm man weitestgehend kommentarlos zur Kenntnis. ${ }^{32}$ Diese Diskussion kann für die Entwicklung Dresdens zur selbsternannten internationalen Fremdenstadt zur Zeit des Kaiserreiches als wegweisend gelten. Das Label beinhaltete nur die Präsenz und die kontinuierliche Rekrutierung der Briten, Amerikaner und Russen, deren mitgebrachter Wohlstand Dresdens Einwohnerschaft, Gewerben und Bildungsanstalten zugutekam, während es Arbeitsmigranten aus Böhmen und anderen Gebieten Österreich-Ungarns nicht umfasste.

Die Unterscheidung der ausländischen Einwohner Dresdens nach Wirtschaftsund Kaufkraft und die damit verbundene bewusste Ausklammerung der ansässigen Österreicher, besonders der Böhmen, erklärt, warum die Selbstzeugnisse von Dresdnern zu ihrer Stadt als Fremdenstadt in diversen Broschüren und Zeitungen diese mit keiner Silbe erwähnten. Denn numerisch gesehen stellten Angehörige der österreichisch-ungarischen Monarchie die meisten Ausländer in Dresden. Von 1871 bis 1910 stieg deren Zahl von 2695 auf 24 386, während die anglo-amerikanische Kolonie laut den statistischen Mitteilungen nur relativ konstant um die 2000 Mitglieder aufzuweisen hatte und ihren Höhepunkt 1895 mit 1184 Briten und 1095 Amerikanern erreichte. ${ }^{33}$

30 Ebd., S. 79.

31 Ebd., S. 86.

32 Statistiken laut R. Jannasch (Hg.), Mittheilungen des Statistischen Bureaus der Stadt Dresden, Nr. 4A, Teil I (im Folgenden: Statistik I), Dresden 1877, S. 37. Die folgenden neun Staaten stellten jeweils 100 oder mehr Einwohner in Dresden laut der Volkszählung aus dem Jahr 1875: Österreich 4 407, Russland 1 033, Großbritannien 779, USA 584, Schweiz 218, Ungarn 166, Frankreich 148, Italien 147, Niederlande 100.

33 Für die Anzahl der Österreicher vgl. Ralf Richter, Reichsausländer in Dresden zwischen 1871 und 1914, Diplomarbeit, Humboldt Universität Berlin 1996, S. 54. Die 
Natürlich muss man hierzu noch sagen, dass die Dresdner österreichische $\mathrm{Zu}$ wanderer nicht als Ausländer wahrnahmen, da die Mehrheit von ihnen aus den deutschsprachigen Gebieten Nordböhmens stammte. Wie Caitlin Murdock gezeigt hat, bildete das sächsisch-böhmische Grenzgebiet zumindest bis ans Ende des Ersten Weltkrieges eine Einheit und seine Bewohner nahmen sich gegenseitig nicht als fremd war. ${ }^{34}$ Die Dresdner sahen auch Tschechisch sprechende Böhmen nicht zwangsläufig als Ausländer an oder konnten Zuwanderer, die sich kulturell als Tschechen identifizierten, nicht immer erkennen. Tschechisch sprechende Böhmen waren laut einem Gesetz, das 1877 erlassen worden war, verpflichtet, wie Österreicher generell, ihre Kinder an deutschen Schulen anzumelden. Dies führte zu ihrer schnellen Integration in die deutsche Gesellschaft bzw. zu ihrer,Germanisierung', ein Prozess, den der tschechische Lohnarbeiter Wenzel Holek ausführlich in seinen Memoiren beschrieb. ${ }^{35}$ Selbst die Alldeutschen waren sich manchmal nicht sicher, ob ein Böhme in Dresden deutsch oder tschechisch war, und hakten oftmals nach. ${ }^{36}$

Doch der entscheidende Unterschied, der erklärt, warum Kommentatoren die 2000 Anglo-Amerikaner in ihren Beschreibungen Dresdens als Fremdenstadt speziell hervorhoben, während sie die mehr als 20000 Böhmen mit keiner Silbe erwähnten, war doch das soziale Gefälle zwischen den beiden Gruppen. Immerhin gehörte Holek wie die Mehrzahl seiner Landsleute in Dresden der Arbeiterklasse an, während die Engländer und Amerikaner, wie der schon erwähnte Zahnarzt Dr. Jenkins, der auch das sächsische Königshaus behandelte, zur wohlhabenden Oberschicht zählten. Kommunale Eliten warben die wohlhabenden Anglo-Amerikaner aktiv an und konstruierten positive Auffassungen von Kosmopolitismus auch nur auf diese Gruppe Ausländer bezogen. Anders ausgedrückt war Ausländer nicht gleich Ausländer. Die Dresdner sahen Begegnungen mit böhmischen Arbeitern nicht als Ausdruck Dresdens als Fremdenstadt oder gar Weltstadt an, aber Begegnungen mit Briten und Amerikanern halfen im Aufbau und bestätigten diese städtische Identität.

Zahlen der ansässigen Amerikaner und Briten entstammen einer 1908 gedruckten Tabelle, die die in sächsischen Städten gemeldeten Subjekte beider Nationen für die Jahre 1895, 1900 und 1905 notierte. Vgl. Die Volkszählung vom 1. Dezember 1905, in: Zeitschrift des K. Sächsischen Statistischen Landesamtes 1 (1908), S. 10.

Vgl. Caituin Murdock, Changing Places. Society, Culture, and Territory in the Saxon-Bohemian Borderlands, 1870-1946, Ann Arbor 2010.

35 Wenzel Holek, Vom Hausarbeiter zum Jugenderzieher. Lebensgang eines deutschtschechischen Handarbeiters, Jena 1921, S. 2 f. Holek schrieb: Die Germanisierung der Kinder vollzieht sich in dieser Volksschicht ganz natürlich und sicher. Mutter und Vater reden die Kinder in ibrer Muttersprache an, diese antworten aber in der Sprache, die sie auf der Straße und in der Schule hören und lernen, also auf deutsch. Auf Seite 25 schilderte Holek auch einen Vorfall mit einem Dresdner Fabrikbesitzer, der nicht glauben wollte, dass Holek Tscheche war. Der Fabrikant war überzeugt, dass Holek deutsch wäre.

36 Stadtarchiv Dresden, Brief von E. R. an Hopf, mit Ausschnitt vom „Dresdner Anzeiger“ vom 7. September 1911, Bestand: Alldeutscher Verband, Signatur 13.1, Akte 38, fol. 103. 


\section{Der Verein zur Förderung Dresdens und des Fremdenverkehrs}

Die Aktivitäten des Dresdner Fremdenverkehrsvereins sind der beste Ausdruck genau dieses limitierten Verständnisses der Internationalität, denn er baute Dresdens Identität als Fremdenstadt beginnend in den 1870er-Jahren aktiv auf und aus. Das Statistische Bureau hatte Zahlen und wirtschaftliche Daten zu den Ausländern in Dresden gesammelt, diese ausgewertet und darauf basierend die Empfehlung erlassen, wohlhabende Migranten aufgrund dieser Daten gezielt anzuwerben. Der im Juni 1875 gegründete „Verein Einheimischer und Fremder zur Wahrung gegenseitiger Interessen“ (der erste in Deutschland überhaupt; 1895 umbenannt in „Verein zur Förderung Dresdens und des Fremdenverkehrs“) übernahm dann die Verantwortung dafür, ausländische Besucher zu rekrutieren, mit der Hoffnung, einige von ihnen zum temporären oder auch ständigen Verbleib zu gewinnen, oder wie der Verein es in seinem zweiten Jahresbericht selbst ausdrückte, um die Fremden nach Dresden zu locken und hier zu fesseln. ${ }^{37}$ Dieser Verein war von Dresdens bürgerlicher und gebildeter Elite geprägt. Im Gesamtvorstand vertreten waren unter anderem drei Akademiker, ein Kollegienrat, ein Advokat, ein Hofkunsthändler, ein Konsul, ein Buchhändler und ein Hoflieferant. Wie auch die Fremdenstudie gaben diese Männer an, den Verein in Würdigung der wirthschaftlichen Bedeutung der Fremdenfrage gegründet zu haben..$^{38}$ Von Beginn an stellte sich der Verein nur in die Dienste wohlhabender Ausländer und betonte schon 1878, dass man Gewerbsgehilfen, Dienstboten, u.s.w. nicht in unserem Sinne als Fremde anzusehende Personen in Betracht zog. Der Verein war an consumtionsfähigen, woblhabenden Fremden, namentlich an Amerikanern und Engländern interessiert. ${ }^{39}$ Genau wie das Statistische Bureau klammerte der Verein die Mehrheit aller Ausländer in Dresden aus - Vertreter der Arbeiterklasse, die meist aus der Donau-

37 Zitat: Zweiter Rechenschafts-Bericht des Vereins Einheimischer und Fremder zur Wahrung gegenseitiger Interessen (eingetragene Genossenschaft) zu Dresden pro 1877, Dresden 1877, S. 5. Vgl. auch Vierunddreissigster Rechenschafts-Bericht des Vereins zur Förderung Dresdens und des Fremdenverkehrs in Dresden, erstattet auf die Zeit vom 1. April 1909 bis 31. März 1910, Dresden 1910, S. 18. In diesem Jahresbericht gab der Verein an, der erste seiner Art in Deutschland gewesen zu sein. Mit ein Auslöser für die Gründung 1875 war wohl ein internationaler Vorfall, der sich im Vorjahr am Dresdner Hauptbahnhof ereignet hatte. Im Mai 1874 hatten Dresdner Polizisten einen englischen Studenten und einen tauben englischen Offizier im Ruhestand am Dresdner Bahnhof nach einem kleinen, wohl der Taubheit geschuldeten Missverständnis verhaftet. Mehrere Engländer und Amerikaner beobachteten diese Auseinandersetzung, und sie wurde sowohl in der Dresdner als auch in der englischen Presse eingehend besprochen und vom Vater des Studenten selbst dem britischen Außenminister zur Klärung vorgelegt. Vgl. National Archives of the United Kingdom, Kew, Christopher S. Penny an den Earl of Derby, HM's Principal Secretary of State for Foreign Affairs, 25. Juni 1874, FO 215-33.

Dritter Rechenschafts-Bericht des Vereins Einheimischer und Fremder zur Wahrung gegenseitiger Interessen, Dresden 1878, S. 3. Ebd., S. 6. 
monarchie kamen - und konzentrierte sich anstatt und gezielt auf wohlhabende, vorwiegend Englisch sprechende Eliten.

Die Jahresberichte des Vereins zeigen eindeutig einen immer stärker werdenden Fokus auf Briten und Amerikaner. Hatten die Russen die Briten und Amerikaner 1875 zahlenmäßig noch übertroffen (die Volkszählung hatte in dem Jahr 1033 offiziell gemeldete Russen gegenüber 779 Briten und 584 Amerikanern ergeben), waren die drei Nationen fünf Jahre später mit 679 britischen, 654 russischen und 580 amerikanischen Einwohnern relativ gleichstark in Dresden vertreten. 40 Doch trotz der Anwesenheit von noch über 600 Russen schilderte der Verein ausschließlich den Rückgang dieser Fremdenkolonie in den Jahresberichten nach 1880 und stellte die Rekrutierung und Präsenz der immer mehr werdenden englischsprachigen Zuwanderer in den Vordergrund: Unter den nabezu 1250 Personen, welche im Laufe dieses Jabres unser Bureau frequentierten, war, wie alljäbrlich die britische und amerikanische Nation am zablreichsten vertreten, wäbrend die in früheren Jabren so stark vertretene russische Nation noch immer zu den selteneren Erscheinungen gehört. ${ }^{41}$ Ein Jahr später hieß es: Die russische Colonie, welche vor Jahren so zablreich war, ist namentlich in Folge des ungünstigen Standes der russischen Währung mehr und mehr zusammengeschmolzen. ${ }^{42}$ Anders sah

40 Zahlen für 1875: Statistik I (wie Anm. 32), S. 37. Zahlen für 1880: Sechster RechenschaftsBericht des Vereins Einheimischer und Fremder zur Wahrung gegenseitiger Interessen, Dresden 1881, S. 8. Man muss hier noch hinzufügen, dass Staatsangehörigkeit bis ins frühe 20. Jahrhundert für viele Menschen eine verwirrende Kategorie darstellte, wie sächsische Beamte selbst 1908 noch feststellten: Die Frage nach der Staatangebörigkeit wird erfabrungsgemäß von der Bevölkerung, der die einschlägigen Rechtsverbältnisse nicht genügend bekannt sind, häufig unrichtig beantwortet. Siehe die Volkszählung vom 1. Dezember 1905 (wie Anm. 33), S. 8, Fußnote 1. Deswegen sind all diese Angaben wahrscheinlich niedriger als die wirklichen Zahlen, da zu vermuten ist, dass einige ausländische Befragte ihres ständigen Wohnortes in Dresden wegen wohl Sachsen als Zugehörigkeit angegeben haben.

41 Fünfter Rechenschafts-Bericht des Vereins Einheimischer und Fremder zur Wahrung gegenseitiger Interessen zu Dresden, erstattet für das Jahr 1880, Dresden 1880, S. 7.

42 Sechster Rechenschafts-Bericht (wie Anm. 40), S. 8. Die russische Kolonie hatte ihren Höhepunkt in den 1870er-Jahren erreicht, und die noch heute bestehende RussischOrthodoxe Kirche wurde zwischen 1872 und 1874 gebaut und eingeweiht. Vgl. Valerija Schälke, Die Russisch-Orthodoxe Kirche in Dresden und ihre Gemeinde, in: Dresdner Hefte 74 (2003), S. 72-77. Diese Ausgabe der „Dresdner Hefte“ ist dem Thema „Rußland und Sachsen in der Geschichte“ gewidmet. Dresdens prominentester russischer Einwohner war der Autor Fyodor Dostoyevsky von 1869 bis 1871 gewesen. Vgl. Erhard Hexelschneider, Meine Adresse: Allemagne, Saxe, Dresden à Monsieur Théodore Dostoiewsky, poste restante, in: Sächsische Heimatblätter 42 (1996), Heft 5, S. 316-321; und Ders., Russische Dichter in Dresden und Leipzig, in: Dresdner Hefte 74 (2003), S. 48-55. Die russische Kolonie erlebte ein kurzes Wiederaufleben infolge des Revolutionsjahres 1905, aber dies war nur ein kurzzeitiges Phänomen. Der Fremdenverkehrsverein schätzte die Lage folgendermaßen ein: Bei den Wirren im Jabre 1906/07 sind viele russische Familien, besonders aus den russischen Ostseeprovinzen, nur deshalb nach Dresden gekommen und haben nur deshalb viele Monate bindurch sich hier aufgehalten, weil sie in ibrer Heimat einen genügenden Schutz für die Sicherheit ibres Lebens nicht mebr zu finden glaubten. Nach der Wiederherstellung geordneter Ver- 
es bei den Engländern und Amerikanern aus. Im Jahr 1882 vermeldete der Verein, wie gewöhnlich war auch dies Jahr die britische und amerikanische Nation bei weitem die überwiegende, die die Dienste des Vereins in Anspruch genommen hatte. ${ }^{43}$ Und vier Jahre später verkündete er sogar freudvoll, dass es Vertreter der britischen und amerikanischen Nation waren, welche bekanntermassen seit vielen Jabren das stärkste Contingent zu unserer Fremdenkolonie stellen und eifrige Freunde unseres Vereins geworden sind. ${ }^{44}$

Diese Freundschaft nährte der Verein mit seinen Aktivitäten. Schon 1880 gab er eine kostenlose 36-seitige Broschüre auf Deutsch und auf Englisch heraus, den „Dresdner Wegweiser, den Fremden gewidmet vom Verein Einheimischer und Fremder zur Wahrung gegenseitiger Interessen“, der Dresdner Sehenswürdigkeiten beleuchtete und Ausländern auch rechtlichen Beistand zusicherte, sollte es zu Disputen mit lokalen Wirten oder Geschäftsinhabern kommen. Man konnte ihn im Informationsbureau des Vereins, in Dresdner Gaststätten und Konsulaten, in vornehmen Kurorten und sogar auf den transatlantischen Schiffen der „HamburgAmerikanischen Packetfahrt-Actien-Gesellschaft" finden. ${ }^{45} \mathrm{Im}$ selben Jahr war auch ein Mitglied der anglo-amerikanischen Kolonie, ein Dr. Charles Eales, in den Gesamtvorstand gewählt worden. ${ }^{46}$ Eine sogenannte Fremdenliste mit Adressen, in der Hauptsache die englische und amerikanische Colonie betreffend, stellte der Verein 1884 zusammen, um es seinen Mitgliedern zu ermöglichen, Werbung und Visitenkarten direkt an englische und amerikanische Einwohner zu schicken. ${ }^{47}$ Diese und ähnliche Publikationen (z. B. die 1910 erschienene Broschüre „Dresden. The Jewel of Northern Europe") sowie weitere Bestrebungen verfolgte der Verein über die nächsten vier Jahrzehnte, was auch die lokale Presse zur Kenntnis nahm. Selbst die recht konservativen „Dresdner Nachrichten“ druckten 1908 einen wohlwollenden Bericht zu den Aktivitäten des Vereins: Der Verein zur Förderung Dresdens und des Fremdenverkehrs hat in der letzten Zeit, teils aus eigenen Mitteln, teils gemeinsam mit der Sektion Dresden des Internationalen Hotelbesitzervereins bedeutende Summen aufgewendet, um auf unsere schöne Stadt, ibre berühmten Kunstsammlungen und ibre sonstigen hervorragenden Einrichtungen in der auswärtigen Presse aufmerksam zu machen. So bat der Verein große Inserate sowie redaktionelle Artikel in den größten deutschen, englischen und amerikanischen Zeitungen veröffentlicht, die geeignet sind, unsere Stadt bei

hältnisse in Russland waren diese Familien wieder in ihre Heimat zurückgekehrt. Vgl. Dreiunddreissigster Rechenschafts-Bericht (wie Anm. 4), S. 4.

43 Siebenter Rechenschafts-Bericht des Vereins Einheimischer und Fremder zur Wahrung gegenseitiger Interessen, Dresden 1882, S. 6.

44 Elfter Rechenschafts-Bericht des Vereins zur Förderung des Fremdenverkehrs in Dresden, Dresden 1886, S. 5 f.

45 Fünfter Rechenschafts-Bericht (wie Anm. 41), S. 5.

46 Ebd., S. 7.

47 Neunter Rechenschafts-Bericht des Vereins Einheimischer und Fremder zur Wahrung gegenseitiger Interessen zu Dresden, erstattet für das Jahr 1884, Dresden 1884, S. 5. 
Nadine Zimmerli

den Fremden wiederbolt in Erinnerung zu bringen und zu einem Besuche Dresdens anzuregen. ${ }^{48}$

Am vielsagendsten ist wohl, dass der Verein 1908 Zweigstellen in London und New York, aber nirgendwo sonst eröffnete. ${ }^{49}$ Der Verein sah die Amerikaner und Briten - als eine große Englisch sprechende Nation wahrgenommen - als seine zahlreichste, sichtbarste und wichtigste Kundschaft und richtete seine Aktivitäten auf ihre Wünsche und Bedürfnisse aus.

Zusammengefasst fingen Dresdens bildungsbürgerliche Eliten, allen voran der Fremdenverkehrsverein, in den 1870er-Jahren gezielt damit an, Dresdens Ruf als Fremdenstadt zu festigen und weitläufig auszubauen, und diese Identität drehte sich zuallererst um die ansässige anglo-amerikanische Kolonie und die alljährlichen abertausenden britischen und amerikanischen Besucher, die es konstant zu werben galt. ${ }^{50} \mathrm{Um}$ die Jahrhundertwende hatte sich diese auf den Anglo-Amerikanern in Dresden basierende Identität dann verfestigt und der Sprung von der Fremden- zur Weltstadt war ein scheinbar kurzer. Bereits 1898 klopfte sich der Verein selbst auf die Schulter, als er angesichts des fünfundzwanzigjährigen Regierungsjubiläums des sächsischen Königs in einer selbstgefälligen Huldigung bemerkte: erst unter der friedengesegneten Regierung des Königs Albert hat Dresden die Bedeutung einer Weltstadt erreicht. ${ }^{51}$ Nach der Reichsgründung hatte Dresden international nicht etwa an Boden verloren, wie man Mitte der 1870er-Jahre noch befürchtet hatte, im Gegenteil, es war sogar zur Weltstadt aufgestiegen, und der Fremdenverkehrsverein trug in seinem Selbstverständnis daran natürlich großen Anteil.

\section{Weltstadt ja oder nein?}

So ganz übertrieben waren die Aussagen des Fremdenverkehrsvereins jedoch nicht. Die Anglo-Amerikaner beschrieben Dresden in ihren Publikationen auch

48 Der Verein zur Förderung Dresdens und des Fremdenverkehrs, in: Dresdner Nachrichten, 20. Mai 1908.

49 Dreiunddreissigster Rechenschafts-Bericht (wie Anm. 4).

50 Thomas St. John Gaffney, amerikanischer Generalkonsul in Dresden von 1905 bis 1913, behauptete, dass jedes Jahr allein 30000 amerikanische Touristen Dresden besuchen würden und sich in der Stadt sehr willkommen geheißen fühlten. Vgl. A prominent place, in: Daily Record, 14. Januar 1910, S. 3. Im Originaltext: Dresden is visited by about 30,000 Americans annually and has a large American colony, which is the highest test of popularity. I do not hesitate to say that the municipal government of the Saxon capital, the cleanliness of its streets and the courteous attitude of the officials and the Dresden public to the visiting Americans are beyond all praise.

51 Vorwort in: Dresden. Eine Fremdenstadt mit ihren Schönheiten in Kunst und Natur. Seiner Majestät dem König Albert von Sachsen am Tage seines fünfundzwanzigjährigen Regierungsjubiläums ehrfurchtsvoll gewidmet vom Pressausschusse des Vereins zur Förderung Dresdens und des Fremdenverkehrs, hrsg. vom Vorsitzenden Rudolf Sendig, Leipzig 1898. 
als cosmopolitan, also als weltstädtisch. Bereits 1873 nannte ein Amerikaner Dresden den attraktivsten deutschen Aufenthaltsort für reisende Amerikaner und auch alle anderen Nationen, dessen Sehenswürdigkeiten der Stadt einen hochangesebenen und kosmopolitischen Ruf verliehen haben und dieser Ruf Dresden zu einem reizenden Ort für kurzzeitige Residenz machte..$^{52}$ Fast vierzig Jahre später hieß es dann 1911 im „Stranger’s Guide“, der englischen Wochenzeitung Dresdens, dass die neugegründete Society for International Friendship den bereits existierenden weltstädtischen Charakter Dresdens (further emphasizing the delightful cosmopolitan spirit already existing) noch unterstreichen würde. ${ }^{53}$ Und 1907 beschrieb der Leitartikel im „Daily Record“, der 1906 gegründeten englischen Tageszeitung Dresdens - und der einzigen englischen Tageszeitung in ganz Deutschland - den Lebensstil der Anglo-Amerikaner als cosmopolitan residence. ${ }^{54}$ Im Selbstempfinden genau jener Gruppe, auf der die Fremdenstadtidentität basierte, hatte Dresden also durchaus weltstädtische Züge, und diese wurden über verschiedene Jahrzehnte hinweg attestiert. ${ }^{55}$

Wie also sind die kosmopolitischen Bestrebungen Dresdens zu bewerten? $\mathrm{Zu}$ gegebenermaßen war das Konzept der kosmopolitischen Fremdenstadt, wie es die Dresdner selbst verstanden, ein stark begrenztes. Es betraf nur einen kleinen Teil der Ausländer der sozialen Ober- und Mittelschicht und war von Anfang an mit Untertönen des lokalen wirtschaftlichen Nutzens behaftet. Aber es ist trotzdem bedeutsam, dass eine Stadt wie Dresden, deren Eliten von der Geschichtswissenschaft oft als stark konservativ und nationalistisch eingeschätzt worden sind, sich selbst als Weltstadt wahrnehmen wollte und zumindest von der Zielgruppe auch so wahrgenommen wurde. Ausländer - vor allem die Anglo-Amerikaner und unter ihnen besonders jene mit ständigem Wohnort in Dresden - waren für das Selbstverständnis der Stadt eindeutig von großer Wichtigkeit.

Diese Fokussierung auf ein bestimmtes Segment Ausländer lässt sich am einfachsten mit innerdeutscher Rivalität erklären. Internationale Anziehungskraft zog auch nationale Bedeutsamkeit nach sich, und in beiden Kategorien befand Dresden sich im Wettstreit mit anderen Großstädten, der neuen Hauptstadt Berlin, aber auch München, Hamburg, Stuttgart, Wiesbaden und Kurorten wie

52 Pennsylvania State University Libraries, [An American Resident], Dresden. Its Attractions and Characteristics, unveröffentlichtes Manuskript, New York 1873, S. 1.

53 Society for International Friendship, in: Stranger's Guide to Dresden, 6. Mai 1911, S. 13.

54 A Merry Christmas, in: Daily Record, 25. Dezember 1907, S. 1.

55 Die Erfahrungen der Amerikaner und Briten in Dresden zur Zeit des Kaiserreiches konkret zu beleuchten, übersteigt den Rahmen dieses Artikels. Ich beschreibe die anglo-amerikanische Kolonie eingehend in meiner Dissertation: Nadine ZimmerLI, "The Rendezvous of All Nations“. Cosmopolitan Encounters in the German City of Dresden before World War I, Diss. University of Wisconsin-Madison 2011, besonders Kapitel 1 bis 3. Eine Skizze der amerikanischen Kolonie ist zu finden in Dies., Die amerikanische Kolonie in Dresden vor 1914, in: Dresdner Hefte 126 (2016), S. 39-50. Die englische Kolonie wurde beschrieben von Ralf Richter, Die Geschichte der ,Englischen Kolonie‘ in Dresden, in: ebd. 70 (2002), S. 47-55. 
Baden-Baden, die allesamt anglo-amerikanische Kolonien besaßen. ${ }^{56}$ München und Stuttgart, auch beide Residenzstädte, gehörten ihrer Größe wegen zu Dresdens engsten Rivalen. Doch spätestens 1908 soll Stuttgart seine einst florierende englisch-sprachige Kolonie verloren haben. ${ }^{57}$ München konnte in touristischen Angelegenheiten ebenfalls überboten werden, auch wenn die bayerische Hauptstadt Dank ihrer Kunstakademie genau wie Dresden viele Amerikaner anzog. ${ }^{58}$ Wie der Fremdenverkehrsverein für das Jahr 1907 süffisant berichtete, hatten die Dresdner Polizeibehörden 482249 Besucher in diesem Jahr verzeichnet, München jedoch nur 452852.59

Selbst die nationalgesinnten Elemente Dresdens werteten internationale Bedeutsamkeit als wichtigen Pluspunkt im innerdeutschen Wettstreit. Die konservativen „Dresdner Nachrichten“ publizierten ebenfalls den Fakt, dass München 1907 weniger Besucher verzeichnet hatte als Dresden, und urteilte stolz: so steht Dresden in Deutschland binsichtlich des Fremdenverkebrs von allen Städten an zweiter Stelle und wird nur noch von Berlin übertroffen. ${ }^{60}$ Und selbst im Wettstreit mit Berlin stellten die Fremdenkolonien Dresdens eine Trumpfkarte für die Zeitung dar. Ein Artikel, der 1905 auf der Titelseite erschien, verkündete poetisch: Ein so farbenbuntes Bild, wie hente die Prager Straße, der Altmarkt, die Wilsdruffer [...], Scbloßstraße und andere bieten, kann so leicht keine zweite deutsche Großstadt aufweisen; auch Berlin nicht; schon deßhalb nicht, weil ibm nur die Reichsfarben schwarz-weiß-rot und die preußischen schwarz-weiß für die Fabnen zur Verfügung stehen (die Berliner Stadtfarben sind dieselben wie die des Reiches), während uns außer den Reichsfarben noch grün-weiß und schwarz-gelb für die Ausschmückung gegeben sind, nach physiologischen Gesetzen gibt das ein schein-

56 Detaillierte Studien existieren für Baden-Baden und Hamburg. Vgl. Ursula Perkow, Residents and Visitors. Die englisch-amerikanische Gemeinde in Baden-Baden, BadenBaden 1990; und Anne D. Petersen, Die Engländer in Hamburg, 1814-1914, Hamburg 1993. Amerikaner und Engländer studierten auch in altehrwürdigen Universitätsstädten wie Göttingen, Heidelberg und Leipzig. Vgl. für die Amerikaner Anja Werner, The Transatlantic World of Higher Education. Americans at German Universities, 17761914, New York 2013; und für die Engländer Thomas Weber, Our Friend „The Enemy“. Elite Education in Britain and Germany before World War I, Stanford 2008. Es ist wichtig zu erwähnen, dass die Anzahl der Amerikaner in Dresden die ihrer Landsleute in diesen Universitätsstädten bei Weitem übertraf. Zum Beispiel studierten 1877 ungefähr nur 25 bis 30 Amerikaner in Göttingen, aber 1875 lebten schon mehr als 584 Amerikaner in Dresden. Im Jahr 1895 lebten 556 Amerikaner in Leipzig aber mehr als 1000 in Dresden.

57 The complaint is made, in: Daily Record, 12. September 1908, S. 3.

58 Vgl. Susanne Böller, American Artists at the Academy of Fine Arts in Munich, 1850-1920, in: Christian Fuhrmeister/Hubertus Kohle/Veerle Thielemans (Hg.), American Artists in Munich. Artistic Migration and Cultural Exchange Processes, Berlin 2009, S. 43-56.

59 Zweiunddreissigster Rechenschafts-Bericht des Vereins zur Förderung Dresdens und des Fremdenverkehrs in Dresden, erstattet auf die Zeit vom 1. April 1907 bis 31. März 1908, Dresden 1908, S. 5 f.

60 Dresdner Nachrichten, 26. Juni 1908, S. 2. 
bar vierfach bunteres Bild. Dazu kommt aber noch das internationale Element der Fremdenstadt Dresden. In der Prager Straße findet sich neben Strips und Stars der Union nicht weniger oft der etwas an die Piratenflagge erinnernde Union-Jack, die alle Farben und noch einige mehr aufweisende Fabne Schweden-Norwegens, daneben der Danebrog, das rot und grün umrabmte Kreuz von Savoyen; sie alle und zablreiche andere geben gerade dieser Hauptverkebrsader ein großstädtisch-internationales Festkleid. 61

Zwar beschwerten sich die britischen Einwohner Dresdens bei dem Verleger der Zeitung über die Beschreibung des Union Jacks als Piratenflagge, einen Vorfall, den sogar der ständige britische Vertreter in Dresden an seine Regierung weitergab, doch drückt der Artikel auch den Stolz der nationalen Elemente auf die Fremdenstadt Dresden aus. ${ }^{62}$ Berlin war monoton und farblos, Dresden hingegen wegen seiner ansässigen Ausländer, allen voran den Amerikanern und den Briten, prachtvoll, bunt und international wichtig. Lokalstolz mischte sich mit internationalem Flair, um die sächsische Hauptstadt über ihren preußischen Rivalen zu stellen. Kulturelle Symbole - besonders die Identität als Fremdenstadt - nahmen für die Dresdner an Wichtigkeit zu, selbst für die konservativsten unter ihnen, als ihre Stadt fast alle politische Macht an Berlin verloren hatte. Internationalität brachte Dresden Pluspunkte und hob die Stadt hervor.

Generell unterstützten die Dresdner die Fremdenstadtidentität. Selbst der als konservativ geltende Oberbürgermeister Beutler fungierte ab 1901 als Ehrenvorsitzender des Fremdenverkehrsvereins und die Stadt subventionierte die Aktivitäten des Vereins auch finanziell. ${ }^{63}$ Beutler war zudem der einzige Oberbürgermeister Deutschlands, der im Gremium des 1905 gegründeten „Deutsch-Englischen Verständigungs-Komitees“ saß, einer internationalen Vereinigung, die darauf abzielte, die wachsenden internationalen Spannungen zwischen Deutschland und Großbritannien aktiv zu reduzieren. ${ }^{64}$ Dazu kommt noch, dass Beutler nicht nur Festreden auf Nationalfeiern hielt. Er eröffnete auch internationale Veranstaltungen, wie z. B. den 4. Internationalen Esperantokongress, der 1908 in Dresden stattfand. ${ }^{65}$ Vom Kerngedanken her konnte diese Bewegung kosmopolitischer

61 Dresden im Flaggenschmuck, in: Dresdner Nachrichten, 26. Mai 1905, S. 1.

62 National Archives of the United Kingdom, Kew, Lord Gough an Marquess of Lansdowne, 1. Juni 1905, FO 30-323.

63 Sechsundzwanzigster Rechenschafts-Bericht des Vereins zur Förderung Dresdens und des Fremdenverkehrs in Dresden, erstattet auf die Zeit vom 1. April 1901 bis 31. März 1902, Dresden 1902, S. 4.

64 Vgl. Gerald Deckart, Deutsch-Englische Verständigung. Eine Darstellung der nichtoffiziellen Bemühungen um eine Wiederannäherung der beiden Länder zwischen 1905 und 1914, Diss. Universität München 1967, S. 39.

65 Über 2000 Esperantisten aus 40 Nationen tagten vom 16. bis 22. August 1908 in der Stadt. Von Dresdens Ruf als Fremdenstadt angezogen, war dies der erste und auch einzige internationale Esperantokongress, der vor dem Ersten Weltkrieg auf deutschem Gebiet stattfand. Wie die amerikanische Esperanto-Zeitschrift „Amerika Esperantisto. A Monthly Magazine of the International Language Esperanto“ es im Februar 1908 ausdrückte: The occasion will be one which no cosmopolitan should miss, if he can by any 
nicht sein und wurde doch genau wie verschiedene national gesinnte Veranstaltungen finanziell von der Stadt unterstützt. ${ }^{66}$ Vor Beginn dieses Kongresses hatten die Veranstalter die Dresdner auch aufgerufen, die ausländischen Esperantisten zu begrüßen, indem sie ihre Häuser grün beflaggten. Den Presseberichten zufolge haben dann auch sehr viele Dresdner Bürger diesem Wunsch entsprochen. ${ }^{67}$ Sie zögerten also nicht, internationale Bewegungen zu zelebrieren, sondern unterstützten Dresdens Fremden- und Weltstadtambitionen tatkräftig.

Es ist auch wichtig festzuhalten, dass die Ortsgruppe Dresden des Alldeutschen Verbandes weitaus weniger Mitglieder als der Fremdenverkehrsverein zählte. Die Alldeutschen erreichten mit 500 Mitgliedern 1903 ihren Höhepunkt, der Fremdenverkehrsverein hatte im selben Jahr 861 Mitglieder. Der Verein selbst erreichte 1912 seine höchste Mitgliedschaft mit 1426 Anhängern, während die alldeutschen Dresdner zu der Zeit nur noch 342 waren. ${ }^{68}$ Internationale Bemü-

means attend, d. h. kein Weltbürger durfte sich diesen Kongress in Dresden entgehen lassen (Amerika Esperantisto 3, Nr. 1, nicht paginiert). Und Oberbürgermeister Beutler schätzte diese weltbürgerlichen Esperantisten dann sehr positiv ein. Wie er in seiner Eröffnungsrede betonte: In einer Zeit, wo die Nationalität und Eigenart jedes einzelnen Volkes im politischen Leben eine so überaus wichtige Rolle spielt und oft recht scharfe und schwer zu lösende Gegensätze schafft, in einer Zeit, wo andererseits die Mittel des Verkehrs eine ungeabnte Ausdebnung und die Leichtigkeit des Verkebrs eine nie erwartete Höhe erreicht haben, in einer solchen Zeit muß jedes Mittel, welches den Völkern die Kenntnis voneinander und darum die Verständigung untereinander erleichtert, ganz besonders willkommen geheißen werden. Vgl. Bericht. IV. Internationaler Esperanto-Kongress, Dresden 16.-22. August 1908, hrsg. vom Sächsischen EsperantoInstitut Dresden, Dresden 1908, S. 31.

66 Die Stadtverordneten entschieden sich, den Kongress erst mit 2000 und dann sogar mit 3000 Mark zu unterstützen, da er die Fremdenstadtidentität Dresdens bekräftigte, indem er wirtschaftlich begüterte Besucher anzog. Vgl. 23. öffentliche Sitzung vom 9. Juli 1908, in: Sitzungsberichte der Stadtverordneten zu Dresden im Jahre 1908, Dresden o. J., S. 922.

67 Aufruf zur Beflaggung: Dresdner Neueste Nachrichten, 16. August 1908, S. 3: Sonntag, den 16. August beginnt der Esperantoweltkongreß, der über 8 Tage gegen 2000 Fremde in unserer Stadt vereinigt. Diesen gilt es ein herzliches Willkommen in unserer Stadt zu entbieten. Wir ersuchen daher die Bürgerschaft Dresdens, dem Beispiel des Rates unserer Stadt folgend, ibre Häuser während der Kongreßwoche zu beflaggen. Zwei Tage später hieß es dann schon Unsere Stadt steht wabrhaftig im Zeichen des grünen Sterns. Vgl. Internationaler Esperanto-Kongreß, in: Dresdner Neueste Nachrichten, 18. August 1908, S. 3; und das „Salonblatt“ bemerkte nach dem Kongress: In allen Strassen baumelte irgendwo zu einem Fenster eine Esperantofahne heraus. Vgl. Esperanto-Kongress, in: Salonblatt, 29. August 1908, S. 7. Auch die Organisatoren waren zufrieden: Überall begegnete man dem Esperantozeichen, von Privathäusern und Hotelgebäuden winken neben den Flaggen in den Landesfarben der angekommenen Fremden die Flaggen der Esperantisten, ein grüner Stern im weißen Felde. Die Straßbabnwagen tragen, soweit sie die Kongreßgebäude passieren, grüne Sterne mit Esperantoaufschriften. Die Schaufenster der Verkaufsläden sind geschmückt und an vielen Läden liest man die freundliche Einladung: , Oni parolas esperante'. Bericht. IV. Internationaler Esperanto-Kongress (wie Anm. 65), S. 10.

68 Mitgliederzahlen für den Verein zur Förderung Dresdens und des Fremdenverkehrs sind in dessen Rechenschaftsberichten von 1877 bis $1914 \mathrm{zu}$ finden, die man sowohl in 
hungen, besonders das Werben um wohlhabende Ausländer, fand lokal also doch weitaus mehr Zuspruch als die nationalistischen, konkret ausländerfeindlichen Aktivitäten der Alldeutschen.

Gewiss war ,Fremdenstadt' nur eine kommunale Identität, die den Dresdnern zur Verfügung stand. Sie war jedoch eine der ältesten und mit anderen am engsten verflochtenen Identitäten. Das „Salonblatt“ - selbsternannte fübrende illustrierte Wochenschrift Sachsens - brachte 1910 eine Sonderausgabe zum Thema Dresden heraus, in der es alle Aspekte der Stadt beschrieb, darunter Dresden als Gartenstadt, Kunststadt, Ausstellungsstadt, Industriestadt und natürlich auch Fremdenstadt. Die Zeitschrift beschrieb Dresden als eine noch sehr junge Industriestadt, doch das Gegenteil traf auf Dresden als Fremdenstadt zu: Man muß sich immer daran erinnern, daß Dresden schon eine berübmte Fremdenstadt war, als die anderen deutschen Städte erst anfingen, es werden zu wollen. ${ }^{69}$ Diese Aussage verweist sogleich noch einmal auf die innerdeutsche Rivalität in Bezug auf ausländische Besucher und Bewohner, in der Dresden implizit den Spitzenplatz einnahm, und sie hob auch den nunmehr alteingesessenen und wichtigen Status Dresdens als Fremdenstadt hervor. Dresden war schon lange vor anderen deutschen Metropolen ein anerkannter internationaler Standort gewesen und werde es auch bleiben.

Hinzu kommt noch, dass die Ausländer für etliche weitere kommunale Identitäten eine bedeutende Rolle spielten, besonders was die Ausstellungen und vor allem die Kunst betraf. Schon 1877 hatte die Fremdenstudie in Hinsicht auf das Kunstgewerbe in Dresden festgestellt: Von ganz besonderer Wichtigkeit für die Entwicklung der Dresdener Production ist es dass nicht nur die Gewerbe, welche die zum Lebensunterhalt notwendigen Gegenstände des täglichen Bedarfs produciren, von der consumptiven Kraft der Fremdenbevölkerung unterstützt werden, sondern dass die Einkommensverbältnisse der Letzteren insbesondere die Unterstützung der Kunst und Kunstgewerbe ermöglichen [...]. Mebr als jede andere bedarf [...] die Kunstindustrie der Unterstützung eines verständnisvollen und opferwilligen Entgegenkommens der Bevölkerung, deren gebildete Elemente durch die biesige Fremdenbevölkerung beträchtlich vermehrt werden. ${ }^{70}$

Die Ausländer blieben bis 1914 eine der wichtigsten Klientele für Dresdens Galerien, Theater und Oper. Scheinbar hörte man z. B. 1906 dank der unzäbligen jungen Damen aus England und Amerika die in Dresden zu Ausbildungszwecken verweilen nichts außer Englisch im Foyer der Oper, wenn Wagners NibelungenSaga aufgeführt wurde. ${ }^{71}$ Dresdens Prosperität und Ruf als Kunststadt und Dresden als Zentrum der Musik beruhte auf kontinuierlicher ausländischer Unterstützung.

der SLUB Dresden als auch im Stadtarchiv Dresden einsehen kann. Die Zahlen für die Dresdner Ortsgruppe des Alldeutschen Verbandes entstammen der ersten Tabelle im Anhang zu Kolditz, Rolle und Wirksamkeit des Alldeutschen Verbandes in Dresden (wie Anm. 11).

69 Dresden als Fremdenstadt, in: Salonblatt, 1. Oktober 1910, S. 1248.

70 Statistik II (wie Anm. 27), S. 89.

71 Wagner's Ring, in: Dresden Daily, 5. April 1906, S. 2. 
Jedoch muss man auch anführen, dass Dresden als berühmte Fremden- und gar Weltstadt ein flüchtiges und brüchiges Konstrukt war. Die nationalen Rivalitäten zwischen England und Deutschland um 1900 wirkten sich auch auf Dresden aus. Um nur kurz ein Beispiel anzuführen: Im März 1900 schrieb ein Engländer an die „London Times“, dass er und seine Landsleute, die der großen englischen Kolonie in der Stadt angehörten, sich wegen der Entwicklung des Burenkrieges, in dem die Deutschen mit den Buren sympathisierten, quasi einer Schreckensherrschaft (reign of terror) ausgesetzt sahen. Dresdner Kinder bewarfen englische Damen mit Schneebällen, die Wände des Anglo-American Clubs und der englischen Kirche wurden mit roter Farbe und Schimpfwörtern verunglimpft und nachts klingelten Unbekannte an von Engländern bewohnten Pensionen Sturm und rissen deren Türklinken ab. ${ }^{72}$ Andere in Dresden ansässige Engländer schrieben schnell, dass dem nicht so sei, aber die Kontroverse wurde auch von der amerikanischen „New York Times" gedruckt und selbst vom britischen Parlament diskutiert. ${ }^{73}$ Die Engländer waren geschockt, dass sie Anglophobie in einer deutschen Stadt, die sie liebten und in der sie zu Tausenden lebten, erfahren mussten. ${ }^{74}$

Vor dem Ersten Weltkrieg waren die Dresdner jedoch meist sehr darauf bedacht, jede Kritik der Anglo-Amerikaner im Keim zu ersticken, um den Ruf als Fremdenstadt zu bewahren. Als sich Leser 1908 in einem Brief an den „Daily Record" darüber beschwerten, dass Dresdner Händler unterschiedliche Preislisten hätten und Angelsachsen regelrecht ausnehmen würden, untersuchte der Fremdenverkehrsverein sofort die Sache und initiierte das Anbringen von Tafeln an allen Geschäften und Pensionen, die ehrlich wirtschafteten. ${ }^{75}$ Der „Daily Record“ attestierte im selben Jahr auch wieder zunehmende Anglophobie in Dresden, die Engländern das Gefühl gab, wegen der britisch-deutschen Rivalität auch in der sächsischen Hauptstadt erneut unerwünscht zu sein. ${ }^{76}$ Die deutschen Zeitungen rechtfertigten sich sofort, so z. B. die „Dresdner Neuesten Nachrichten“: Im übrigen finden aber Engländer und Amerikaner gerade bier in Dresden sowobl als Mitglieder der Kolonie wie als Touristen das weitgehendste Entgegenkommen und man kann oft in der Öffentlichkeit beobachten, daß der Dresdner Fremden, und

72 E. J. Walker, German Animosity in Dresden. To the Editor of the Times, in: Times (London), 12. März 1900, S. 10.

73 The Dresden Church Outrage, in: New York Times, 17. März 1900, S. 3; Anti-British Demonstrations in Saxony, Mr. Talbot, answered by Mr. Brodrick, House of Commons Debates, March 15, 1900, vol. 80 cc918-9.

74 Wie der Korrespondent der „London Times“ traurig urteilte: Notbing could have given Englishmen resident in Germany more pain than to learn that Dresden, of all German cities, had been the scene of excesses directed against the British colony. Those of us whose work lies amid the politics and din of Berlin, or who tire of the materialism or the dulness of other German towns, have always regarded the delightful capital on the Elbe as an earthly, rest for the weary'. Vgl. [From Our Own Correspondent], Germany, in: Times (London), 17. März 1900, S. 7.

75 Vgl. Daily Record, 17. Mai 1908, S. 5; auch Zweiunddreissigster Rechenschafts-Bericht (wie Anm. 59), S. 12.

76 Local Anglophobia, in: Daily Record, 15. Mai 1908, S. 1. 
gerade Angelsachsen gegenüber, von einer fast übertriebenen Höflichkeit ist. [...] Er [the Daily Record] täte besser daran, der Wabrbeit gemäß zu konstatieren, daß die Bevölkerung und die Presse von Dresden allen Fremden, auch Engländern und Amerikanern gegenüber, so entgegenkommend und freundlich gesinnt sind, wie das sonst in einer andern Stadt Deutschlands der Fall ist. ${ }^{77}$

Der Krieg der Worte verstummte rasch, und die schnelle und ausführliche Rechtfertigung zeigt, dass Dresden um seinen guten Ruf den Anglo-Amerikanern gegenüber besorgt war und ihn versuchte, sofort zu verteidigen. Ein zweiter Artikel des „Daily Record“ stellte dann auch klar, dass er im ersten Artikel nicht die Taten der Dresdner Bevölkerung, sondern die antienglischen Berichte in der deutschen Presse gemeint hatte. ${ }^{78}$

Niemand konnte jedoch den Ausbruch des Ersten Weltkrieges - den man als Triumph nationaler Gesinnungen betrachten kann - auf lokaler Ebene verhindern oder weiterhin rechtfertigen, dass Briten und Amerikaner trotz der politischen Großlage noch willkommen wären. Die anglo-amerikanische Kolonie löste sich zwischen 1914 und 1917 auf, und mit ihr nahm auch Dresdens Ruf als Fremdenstadt ein jähes Ende. Andere Identitäten wie Industriestadt, Kunststadt oder Ausstellungsstadt blieben Dresden nach dem Ende des Krieges 1918 erhalten, nicht jedoch Fremdenstadt. Oberbürgermeister Bernhard Blüher schrieb 1930 einen Beitrag zu „Dresden als Internationale Fremdenstadt“ im Zuge der Zweiten Internationalen Hygieneausstellung. Wie er eingestehen musste: Groß war auch stets der Anteil der amerikanischen Gäste. Zablreiche Ausländer haben in der Vorkriegszeit Dresden zum dauernden Wobnsitz gewäblt, ibre Kolonien gaben hier dem gesellschaftlichen Leben ein besonderes internationales Gepräge. Doch der Weltkrieg beeinträchtigte diese Seßhaftigkeit stark. Auch waren die Verkebrszablen vor dem Weltkriege [...] noch lange nicht wieder erreicht. ${ }^{79}$ Dresdens Tage als international berühmte und frequentierte Fremdenstadt gehörten der Vergangenheit an.

Im Kaiserreich hatte Dresden dank seiner wohlhabenden und einflussreichen ausländischen Einwohner, allen voran den Briten und Amerikanern, zumindest in deren Augen und in den Annalen des Fremdenverkehrsvereins einen weltstädtischen Ruf genossen. Als aus den Einwohnern, die Dresdens Fremdenstadtidentität gestützt und verstärkt hatten, dann 1914 und 1917 Feinde wurden, blieb von der internationalen Bedeutsamkeit Dresdens nicht mehr viel übrig. Dass Dresden trotz seiner Ambitionen nicht auf Dauer zur Weltstadt wurde, heißt aber nicht,

77 Lokale Anglophobie, in: Dresdner Neueste Nachrichten, 20. Mai 1908, S. 2.

78 Local Anglophobia, in: Daily Record, 21. Mai 1908, S. 1. Zur Rolle, die deutsche und britische Zeitungen während der Vorkriegsjahre generell spielten, vgl. DoмINIK GEPPERT, Pressekriege. Öffentlichkeit und Diplomatie in den deutsch-britischen Beziehungen 1896-1912 (Veröffentlichungen des Deutschen Historischen Instituts London 64), München 2007.

79 Bernhard BlüHer, Dresden als Internationale Fremdenstadt, in: Heinrich Zerkaulen (Hg.), Das Deutsche Hygiene-Museum. Festschrift zur Eröffnung des Museums und der Internationalen Hygiene-Ausstellung Dresden 1930, Dresden 1930, S. 11 f. 
dass das sorgfältig kultivierte Fremdenstadtlabel ohne Bedeutung gewesen wäre. Egal wie restriktiv beide Konstrukte - Fremdenstadt und Weltstadt - waren, so gewähren sie doch einen Einblick in die Art und Weise, mit der sich zumindest die Dresdner - die als Repräsentanten einer deutschen Großstadt gelten können - mit der globalisierenden Welt um 1900 auseinandersetzten, und wie sie regionale, nationale und internationale Impulse miteinander verknüpfen konnten.

Die englische Schülerin Kathleen Courtney malte 1896 unbewusst ein metaphorisches Bild der Zusammenwirkung der nationalen und internationalen Elemente der Stadt, die miteinander im wahrsten Sinne des Wortes im Einklang standen. Als sie im sogenannten amerikanischen Viertel (heute Südvorstadt) in ihrem Zimmer saß und einen Brief an ihre Mutter schrieb, hörte sie vaterländische Musik aus dem Restaurant Bergkeller, und immer wenn die Kapelle stoppte, vernahm sie die Orgelmusik und auch den Chor der amerikanischen Kirche St. John's (1884 eingeweiht). ${ }^{80}$ Amerikanischer Chor und deutsche Kapelle existierten neben- und miteinander. Obwohl die amerikanische Musik leiser war, behauptete sie sich und konnte konstant vernommen werden, auch wenn die nationalen Töne doch lauter waren. Deutsche und amerikanische Klänge, die die Engländerin Courtney übrigens laut ihres Briefes beide mochte, vermischten sich in Dresden. So überrascht es am Ende auch nicht, dass ein konservativer Mann wie Oberbürgermeister Beutler sowohl bei nationalen Festen Reden hielt als auch internationale Tagungen wie den Esperantokongress eröffnete - am Ende passten beide ins Selbstverständnis der Stadt und all diese Aktivitäten kamen Dresden zugute. Um noch einmal den Ausspruch der Pensionsleiterin von 1896 in Erinnerung zu rufen, obne die Ausländer wäre Dresden ein Dorf. Und wer will schon Bürgermeister eines Dorfes sein, wenn Bürgermeister einer Weltstadt auch eine Option ist?

80 The Women's Library, London Metropolitan University, Kathleen D'Olier an Mama, 17. Mai 1896, Papers of Kathleen D'Olier Courtney, 7KDC/A/2. 